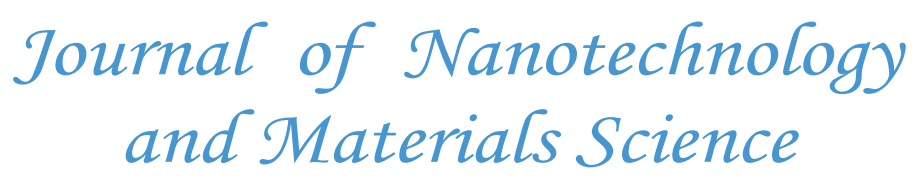

\title{
A Comparison of Unilamellar and Multilamellar Lipoplex- es in Terms of Transfection Efficiency of Human Embryonic Kidney Cells
}

\author{
Mahshid Shokri', Mohmood Tavallaie ${ }^{2 *}$, Seyed Mostafa Hosseini \\ ${ }^{1}$ Department of Biomedical Engineering, Science and Research Branch, Islamic Azad University, Tehran, Iran \\ ${ }^{2}$ Human Genetic Research Centre, Baqiyatallah Medical Sciences University, Tehran, Iran
}

*Corresponding author: Mohmood Tavallaie, Human Genetic Research Centre, Baqiyatallah Medical Sciences University, Tehran, Iran, Tel: (+ 98) 26403292; Fax: (+ 98) 26413382; E-mail: tavalla.mah@gmail.com

\begin{abstract}
Non-viral gene delivery systems have attracted many attentions in the field of gene therapy. Liposome nanoparticles are regarded as one of the most effective non-viral gene delivery vectors. With attention to the fact that one important feature of these carries is their transfection efficiency, in this study we compared the efficiency of unilamellar and multilamellar liposomes in transfecting human embryonic kidney cells by LacZ gene. A liposomal formulation made of DOTAP/DOPE/Cholesterol was fabricated through modified lipid film hydration method. In order to obtain unilamellar lipoplexes extrusion was performed. Lipoplexes were prepared by complexation of liposomes with LacZ gene. Zeta potential, size and polydispersity of lipoplexes and liposomes were measured. In order to determine transfection ability of them, LacZ expression was measured using beta-galactosidase assay. The results show that multilamellar liposomes in spite of their larger sizes have higher transfection compared to unilamellar ones. This is indicative of better performance of multilamellar lipoplexes in gene therapy applications.
\end{abstract}

Received date: September 27, 2016 Accepted date: December 02, 2016 Published date: December 08, 2016

Citation: Tavallaie, M., et al. A Comparison of Unilamellar and Multilamellar Lipoplexes in Terms of Transfection Efficiency of Human Embryonic Kidney Cells. (2016) J Nanotech Mater Sci 3(2): 43- 46 .

DOI: $10.15436 / 2377-1372.16 .1140$

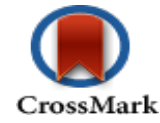

Keywords: Cationic liposome; Lipoplex; Transfection; Gene delivery; Human embryonic kidney cell

\section{Introduction}

Therapeutic gene therapy has offered a useful strategy for treatment of acquired and inherited diseases through transfection of malfunctioning cells by specific defective genes ${ }^{[1,2]}$. Gene therapy could help to correct genetic deficiencies in many genetic diseases including, malignant melanoma, cystic fibrosis and Gaucher's disease ${ }^{[3]}$.

Owing to the fact that the efficiency of naked DNA injection is insufficient for applications, such as DNA vaccination, one of the main challenges in this field is how to deliver nucleic acids to target cells and tissues. Therefore, reliable carrier systems are required. Hence, different viral (include retrovirus, lentivirus, adenovirus, herpes virus, pox virus and so on) and non-viral vectors in addition to modified naked DNA have been introduced to deliver exogenous genes into the cells efficiently ${ }^{[4]}$. Although transfection efficiencies are high in viral vectors, these vectors suffer from many problems such as noticeable immunogenicity leading to induction of inflammatory system, the risk of insertional mutagenesis, limited transgenic capacity, high $\operatorname{cost}^{[4]}$. In this regard, non-viral vectors have advantages over viral vectors in terms of safety, large scale manufacturing and lower prices, albeit their disadvantage especially for in vivo applications is their relatively inferior transfection efficiency compared to viral vectors $^{[5]}$.

Transfection efficiency depends on a number of factors including chemical structure of lipids and supramolecular struc- 
ture and morphology of lipoplexes ${ }^{[3,6]}$, as well as the presence of helper lipids (such as Dioleoyl Phosphatidylethanolamine (DOPE), cholesterol or Dioleoyl Phosphatidylcholine (DOPC) ${ }^{[7]}$ which helps to the association of the DNA with the lipoplexes and transfection efficiency ${ }^{[3,8-10]}$. Liposome size which determines the mechanism of particle entry into cells is another factor affecting transfection efficiency ${ }^{[11]}$. Charge ratio $( \pm)$ of the assembled complexes which could be seen in terms of zeta potential is the other effective factor in efficiency determining the stability of lipoplexes ${ }^{[3,9]}$

In this article, a liposome nanoparticle formulation of DOTAP/DOPE/Cholesterol was fabricated and its transfection was investigated. Both unilamellar and multilamellar liposomes were applied to transfect HEK-293 cells by LacZ gene and their transfection efficiency was compared.

\section{Methods and Materials}

\section{Materials}

1,2-Di-O-Octadecenyl-3-Trimethylammonium Propane (DOTAP) and 1,2-dioleoyl-sn-glycero-3-phosphoethanolamine (DOPE) were purchased from Avanti Polar lipids. Cholesterol and Sucrose were obtained from Sigma-Aldrich and Alfa Aesar, respectively. Human Embryonic Kidney 293 (HEK-293) was obtained from American Type Culture Collection. Dulbecco's Modified Eagle's Medium (DMEM) with 10\% Fetal Bovine Serum (FBS) and penicillin-streptomycin solutions were purchased from GIBCO Inc. Beta-galactosidase and BCA assay kits were purchased from Progma and Pierce, respectively. All the solvent were of analytical grade.

\section{Formulaion of liposomes and lipoplexes}

Cationic liposomes were fabricated using a modified lipid film hydration method. Briefly, DOTAP/DOPE/cholesterol with a molar ratio of 1:1:2 were dissolved in chloroform (total concentration of $5 \mathrm{mg} / \mathrm{ml}$ ). Then, the Solvent was removed by heating the solution at $40^{\circ} \mathrm{C}$ for 6 hours in ambient pressure followed by applying vacuum for 24 hours. The dry lipid film was then hydrated through $1 \mathrm{ml}$ of $15 \mathrm{mg} / \mathrm{ml}$ sucrose aqueous solution to obtain multilamellar vesicles. Unilamellar vesicles were also obtained through extrusion of multilamellar liposome suspension using a mini-extruder using a $100 \mathrm{~nm}$ polycarbonate membrane. Finally, lipoplexes were prepared by mixing DNA and cationic liposomes and incubating for 20 minutes at room temperature.

\section{Particle size and surface charge of lipoplexes}

To ensure the incorporation of $1 \mu \mathrm{g}$ DNA into liposomes with the $( \pm)$ charge ratios of $1.5: 1$, mean particle size of liposomes and lipoplexes were determined using particle size analyzer (Brookhaven Instruments). Furthermore, the surface charge was measured using zeta potential analyzer (Brookhaven Instruments). All the measurements were performed at room temperature using water as the solvent.

\section{Cell Culture and transfection assay}

In order to compare transfection efficiency of both unilamellar and multilamellar lipoplexes, the reporter gene LacZ and HEK-293 cell line were employed. The cells were incubated at $37^{\circ} \mathrm{C}$ and $5 \% \mathrm{CO}_{2}$ in DMEM with $10 \% \mathrm{FBS}$. One day prior to transfection, the cells were seeded into 12-well plates with a cell density of $3 \times 10^{5}$ cells per well. Immediately before the test start, culture medium was replaced with serum-free DMEM. Lipoplexes were directly added to the medium and after 5 hours incubation, the culture medium was replaced by media with serum. LacZ expression was measured 28 hours after transfection using beta-galactosidase assay and normalized with cellular protein measured through BCA assay. LacZ gene encodes the $\beta$-galactosidase enzyme. This enzyme catalyzes the hydrolysis of $\beta$-galactosides into monosaccharides. One such $\beta$-galactoside is X-gal (5-bromo-4-chloro-3-indolyl-beta- D-galactopyranoside), an organic compound. $\beta$-Galactosidase cleaves X-gal into galactose and 5-bromo-4-chloro-3-hydroxyindole; this second compound is then oxidized and final product is blue in color. $\mathrm{X}$-gal staining therefore provides a visual assay of LacZ activity. It should be noted that DNA alone was employed as the negative control in this experiment.

\section{Statistical analysis}

One-way ANOVA followed by Dunnett's multiple comparisons test was performed for the test of statistical significance using Graph Pad Prism software (version 6.00 for Windows, Graph Pad Software, La Jolla California USA, www. graphpad.com)

\section{Results}

\section{Particle size and polydispersity of liposomes and lipoplexes}

(a)

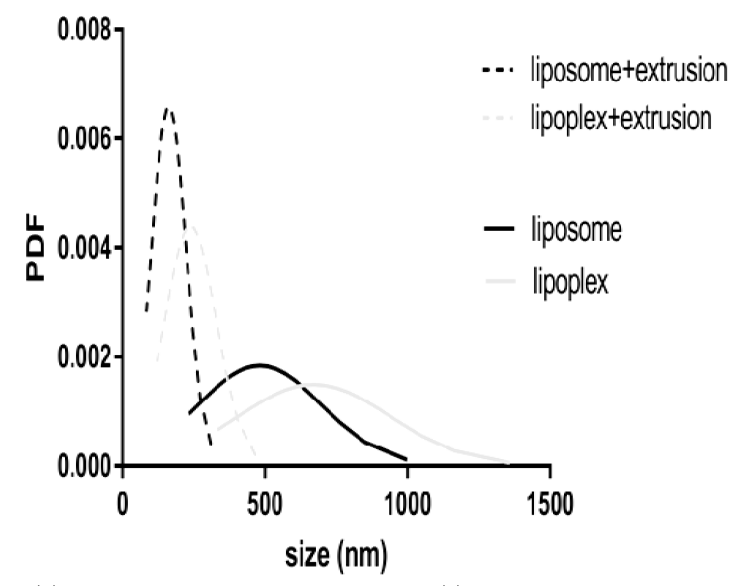

(b)

(c)
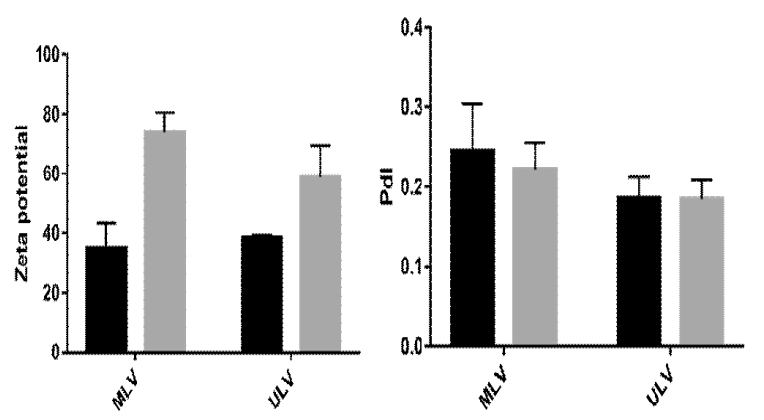

Figure 1: represents particle size, polydispersity and mean zeta potential of lipoplexes compared to the corresponding liposomes. 


\section{Transfection efficiency}

Multilamellar and unilamellar samples were compared in terms of transfection of HEK-293 cells. DNA alone was employed as a negative control in this experiment. The results have been shown in figure 2. It is evident that both liposomal formulations have increased the transfection dramatically compared to the negative control.

\section{Expression per celluar protein}

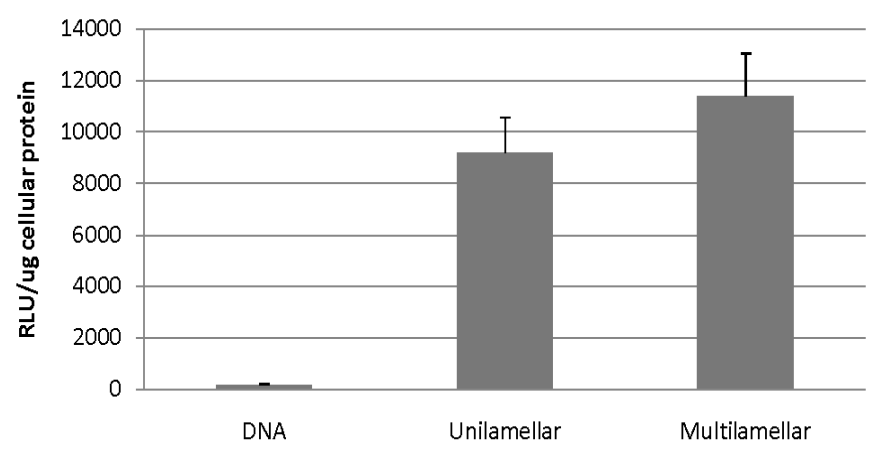

Figure 2: Cellular transfection efficiency of liposomal formulations.

As could be seen in the figure, transfection efficiency for multilamellar vesicles was higher compared to unilamellar ones. However, statistical analysis resulted in a p-value of 0.00034 for MLV and 0.00159 for ULV which shows low presumption against the null hypothesis.

\section{Discussion}

Cationic liposomes of DOTAP/DOPE/Cholesterol were prepared through a modified dry lipid film method. The amphiphilic nature of lipids causes self-assembly in aqueous media and multilamellar vesicles are produced via sonication. Extrusion of multilamellar vesicle suspension in the next step is expected to produce unilamellar vesicles ${ }^{[12]}$.

It could be seen from Figure 1 that the mean particle size of vesicles increases when DNA is added to the liposomes which are in agreement with the data reported by other authors ${ }^{[13,14]}$. Observing the dequenching of the fluorescent probe NBD-PE incorporated along with Rh-PE into the liposomes membrane, Radwan Almofti et al. found DNA-induced liposome-liposome responsible for the change in liposome size, upon addition of $\mathrm{DNA}^{[13]}$. The positive charge of liposomes caused by amine groups of cationic lipid, DOTAP, decreased but remained positive through the addition of DNA which supports the formation of liposome-DNA complexes. The net positive charge of the formulations makes them appropriate for transfection.

In this study, transfection efficiency was ameliorated in both formulations. Enhanced transfection of different cationic liposome-DNA complexes and its mechanism has been the subject of numerous reports ${ }^{[15-18]}$. The improved transfection could be attributed to the net positive charge of vesicles which cause the high efficiency of DNA complexation, electrostatic interaction with negatively charged cell membrane resulting in adsorptive endocytosis ${ }^{[19]}$ and enhanced endosomal escape ${ }^{[20]}$. It has been distinguished that mode of cellular uptake of liposomes is highly dependent of is physicochemical properties ${ }^{[13]}$.

The superiority of multilamellar vesicles over unil- amellar ones has been always controversial. It is also possible that the higher transfection efficiency of multilamellar vesicles compared to unilamellar ones is mainly a result of lipoplexes size rather than liposome type. Ross et al. showed that lipoplexes size is a major determinant of lipofection and the effect of other parameters is mediated through their influence on lipoplexes size $^{[11]}$. They demonstrated that larger lipoplexes lead to greater cellular association and uptake than smaller lipoplexes. It has been also suggested that larger complexes may facilitate cell surface contact by sedimentation while the lower activity of smaller lipoplexes could be due to sedimentation problem ${ }^{[21]}$.

\section{Conclusion}

In his model, transfection of human embryonic kidney cells by LacZ gene was compared in a liposomal formulation of DOTAP/DOPE/Cholesterol in two forms of unilamellar and multilamellar. Modified lipid film hydration method was used to fabricate liposomes and then complexation of liposomes with LacZ gene was performed and Lipoplexes were achieved. Based on results, the size of unilamellar liposomes is obviously smaller than multilamellar ones while zeta potential and polydispersity was slightly decreased in them. After complexation with gene, lipoplexes have increased sizes and polydispersities whether in unilamellar or multilamellar liposomes. Zeta potential of lipoplexes was less than liposomes due to neutralization of positive charge of liposomes with negative charge of gene. The results of measurement of expression of LacZ gene using beta-galactosidase assay showed higher transfection of this gene in multilamellar liposomes compared to unilamellar ones.

\section{References}

1. Ciani, L., Ristori, S., Salvati, A., et al. DOTAP/DOPE and DC-Chol/ DOPE lipoplexes for gene delivery: zeta potential measurements and electron spin resonance spectra. (2004) Biochim Biophys Acta 1664(1): 70-79.

2. Zhang, Y., Satterlee, A., Huang, L. In Vivo Gene Delivery by Nonviral Vectors: Overcoming Hurdles? (2012) Mol Ther 20(7): 1298-1304. 3. Ma, B., Zhang, S., Jiang, H., et al. Lipoplex morphologies and their influences on transfection efficiency in gene delivery. (2007) J Control Release 123(3): 184-194.

4. Nayerossadat, N., Maedeh, T., Ali, P.A. Viral and nonviral delivery systems for gene delivery. (2012) Advanced Biomedical Research 1: 27.

5. Cevher, E., Çağlar, E.Ş., Sezer, A.D. Gene Delivery Systems: Recent Progress in Viral and Non-Viral Therapy. (2012) Intech.

6. Kerner, M., Meyuhas, O., Hirsch-Lerner, D., et al. Interplay in lipoplexes between type of pDNA promoter and lipid composition determines transfection efficiency of human growth hormone in NIH3T3 cells in culture. (2001) Biochim Biophys Acta 1532(1-2): 128-136.

7. Pozzi, D., Marchini, C., Cardarelli, F., et al. Transfection efficiency boost of cholesterol-containing lipoplexes. (2012) Biochim Biophys Acta 1818(9): 2335-2343.

8. Zuhorn, I.S., Bakowsky, U., Polushkin, E., et al. Nonbilayer phase of lipoplex-membrane mixture determines endosomal escape of genetic cargo and transfection efficiency. (2005) Mol Ther 11(5): 801-810.

9. Tros de Ilarduya, C., Sun, Y., Düzgüneş, N. Gene delivery by lipoplexes and polyplexes. (2010) Eur J Pharm Sci 40(3): 159-170.

10. Simberg, D., Weisman, S., Talmon, Y., et al. DOTAP (and Other Cationic Lipids): Chemistry, Biophysics, and Transfection. (2004) Crit Rev Ther Drug Carrier Syst 21(4): 257-317. 
11. Ross, P., Hui, S. Lipoplex size is a major determinant of in vitro lipofection efficiency. (1999) Gene Ther 6(4): 651-659.

12. Dhandapani, N.V., Thapa, A., Sandip, G., et al. Liposomes as novel drug delivery system: A comprehensive review. (2013) Int J Res Pharm Sci 4(2): 187-193.

13. Almofti, M.R., Harashima, H., Shinohara, Y., et al. Cationic liposome-mediated gene delivery: biophysical study and mechanism of internalization. (2003) Arch Biochem Biophys 410(2): 246-253.

14. Moghaddam, B., McNeil, S.E., Zheng, Q., et al. Exploring the Correlation Between Lipid Packaging in Lipoplexes and Their Transfection Efficacy. (2011) Pharmaceutics 3(4): 848-864.

15. Felgner, P.L., Barenholz, Y., Behr, J.P., et al. Nomenclature for synthetic gene delivery systems. (1997) Hum Gene Ther 8(5): 511-512.

16. Lasic, D.D., Barenholz, Y. Handbook of Nonmedical Applications of Liposomes: Theory and basic sciences. (1996) CRC Press.

17. Maurer, N., Mori, A., Palmer, L., et al. Lipid-based systems for the intracellular delivery of genetic drugs. (1999) Mol Membr Biol 16(1): 129-140.

18. Buñuales, M., Düzgünes, N., Zalba, S., et al. Efficient gene delivery by EGF-lipoplexes in vitro and in vivo. (2011) Nanomedicine 6(1): 89-98.

19. Felgner, P.L., Gadek, T.R., Holm, M., et al. Lipofection: a highly efficient, lipid-mediated DNA-transfection procedure. (1987) Proc Natl Acad Sci U S A 84(21): 7413-7417.

20. Lasic, D.D. Liposomes in gene delivery. (1997) CRC press.

21. Boussif, O., Zanta, M.A., Behr, J. P. Optimized galenics improve in vitro gene transfer with cationic molecules up to 1000-fold. (1996) Gene Ther 3(12): 1074-1080. 